Meta

Journal des traducteurs

Translators' Journal

\title{
On the Implausibility of Equivalent Response (III)
}

\section{Qian Hu}

Volume 38, numéro 2, juin 1993

URI : https://id.erudit.org/iderudit/003146ar

DOI : https://doi.org/10.7202/003146ar

Aller au sommaire du numéro

\section{Éditeur(s)}

Les Presses de l'Université de Montréal

\section{ISSN}

0026-0452 (imprimé)

1492-1421 (numérique)

Découvrir la revue

Citer cet article

$\mathrm{Hu}$, Q. (1993). On the Implausibility of Equivalent Response (III). Meta, 38(2), 226-237. https://doi.org/10.7202/003146ar
Résumé de l'article

Dans le but de déterminer si la réponse équivalente constitue une théorie vraisemblable ou non, la troisième partie de cette série d'articles examine de manière plus détaillée le modèle de traduction conçu selon la méthode des trois étapes (analyse, tranfert, restructuration) de Nida. Ce faisant, l'on essaie d'établir s'il est possible d'arriver à une équivalence dynamique dans la pratique de la traduction. 


\section{ON THE IMPLAUSIBILITY OF EQUIVALENT RESPONSE (PART III)}

QIAN HU

Pacific Grove, California, USA

\section{CHAPTER III \\ THREE-STAGE PROCEDURE \\ Résumé}

Dans le but de déterminer si la réponse équivalente constitue une théorie vraisemblable ou non, la troisième partie de cette série d'articles examine de manière plus détaillée le modèle de traduction conçu selon la méthode des trois étapes (analyse, tranfert, restructuration) de Nida. Ce faisant, l'on essaie d'établir s'il est possible d'arriver à une équivalence dynamique dans la pratique de la traduction.

In the previous chapter, the nature of language was analyzed - though not exhaustively - in an attempt to justify the postulation that language is a contradictory congruence. Each language shares with others the presumed characteristics or tendencies which provide a base for inter-lingual communication. However, each is an ad hoc autonomous system of dialectical relationships, which makes impossible the equivalent effect of interlingual communication.

In assessing the principle of equivalent response, it is necessary first to analyze the procedure which Nida devised for obtaining dynamic equivalence. If equivalent effect is assumed to be achievable, it is more clearly demonstrable after analysis whether dynamic equivalence can be reached through this procedure.

\section{A three-stage procedure}

According to Nida (1969b: 33), there are two different systems for translating; one is a single-stage procedure and the other a three-stage procedure. The single-stage procedure is represented as follows:

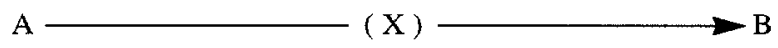

Figure 3

In this diagram, " $\mathrm{A}$ " stands for the source language and " $\mathrm{B}$ " for the target language. "(X)" represents "any intermediate structure which may have been set up as a kind of universal structure to which any and all languages might be related for more economic transfer" (ibid.). In this system, a series of rules are set up to "be applied strictly in order" and "to specify exactly what should be done with each item or combination of items in the source language so as to select the appropriate corresponding form in the receptor language" (ibid.). This approach is based on the application of rules to the surface structure of language. Nida (1969b: 33) argues that this system is inadequate, since it cannot accurately represent the procedure of good translation. Nida (1969b: 33-34) believes that the alternative system of translation is much better. It consists of an elaborate procedure comprising three stages - analysis, transfer, and restructuring. This is represented in Figure 4. 




Figure 4

This approach, although more cumbersome, can "reflect much better the real nature of linguistic structures, and therefore can reflect much more accurately what happens in good translation and represent a much more efficient method for the mastery of translation technique than the first system" (Nida and Taber 1969b: 34).

The first stage is analysis; here the process which Nida terms "back-transformation" is employed to analyse the surface structure of the source language in terms of the grammatical relationships ${ }^{1}$. "Back-transformation" is the reduction of the surface structure to its underlying kernels. For Nida, this is one of the most effective ways to handle the surface structure. To determine the underlying relationships between the elements in a phrase or a sentence, it is essential to get beneath the surface structure by the process of back-transformation and determine the kernel from which the surface structure is derived.

To go beneath the surface structure is not merely to "provide the clearest and most unambiguous expression of the relationship. It also makes it easier to transfer the message from the source language to the receptor language." He claims it is easier because "in all languages there are half a dozen to a dozen basic structures out of which all the more elaborate formations are constructed by means of the so-called transformations;" also because "languages agree far more on the level of the kernels than on the level of the more elaborate structures ${ }^{2}$." In addition, a message can be transferred on the kernel level or a near-kernel level with a minimum of distortion (Nida and Taber 1969b: 39).

Transfer, the second stage, is made at a near-kernel level (Nida and Taber 1969b: 3940 , footnote). This takes place in the translator's brain and the actual process is undefined since neurology has failed - as yet - to pinpoint how and where two languages contact or dovetail. This stage, transfer, crucial as it is in the procedure, remains conjectural.

In the final stage, restructuring, "the transferred material is restructured in order to make the final message fully acceptable in the receptor language" (ibid.: 33). In restructuring, problems of style are taken into consideration: "(1) the varieties of language or of styles which may be desirable, (2) the essential components and characteristics of these various styles, and (3) the techniques which may be employed in producing the type of style desired" (ibid.: 120). The formal features of the two languages must be considered in order to produce the receptor language text in a style comparable to that of the original. As the transferred material is on a near-kernel level, the process of restructuring implies the employment of the means of transformations.

\section{Deep structure, surface structure and semantic interpretation}

From the brief observation of Nida's three-stage system for translation, it can clearly be seen that the technique of transformational grammar is applied in the procedure; conversely, the design of the three-stage procedure is based on the theory of transformational grammar. Nida (1964: 60) adopts the Chomskian generative theory as his basic 
view of language, claiming that the generative view of language is particularly important for the translator, for "it provides him first with a technique for analyzing the process of decoding the source text, and secondly with a procedure for describing the generation of the appropriate corresponding expressions in the receptor language." In this way, the translator can "go beyond mere comparisons of corresponding structures" and "describe the mechanisms by which the total message is decoded, transferred, and transformed into the structures of another language" (Nida 1964: 9).

Certain fundamental concepts and issues of transformational grammar which are pertinent to this discussion now need to be clarified.

The application of back-transformations and forward-transformations in the process of translation is intended to secure a transfer of message from the source language to the receptor language "with a minimum of distortion." This results from the belief that the deep structure determines the meaning of a sentence as well as the idea that languages agree far more in deep structure than in surface structure. Without this conviction, Nida would not proceed by such a devious route to arrive at the surface structure of the receptor language. The single-stage procedure is more straightforward and requires less effort. This is also shown by his attention to formal features (elements of style) in the stage of restructuring after the transfer.

Can Nida's conviction be justified? Is the meaning of a sentence determined by the deep structure or the surface structure?

In the theory of transformational grammar, the deep structure of a sentence is constituted by a base P-marker illustrated in the following tree diagram (Jacobsen 1977: 79):

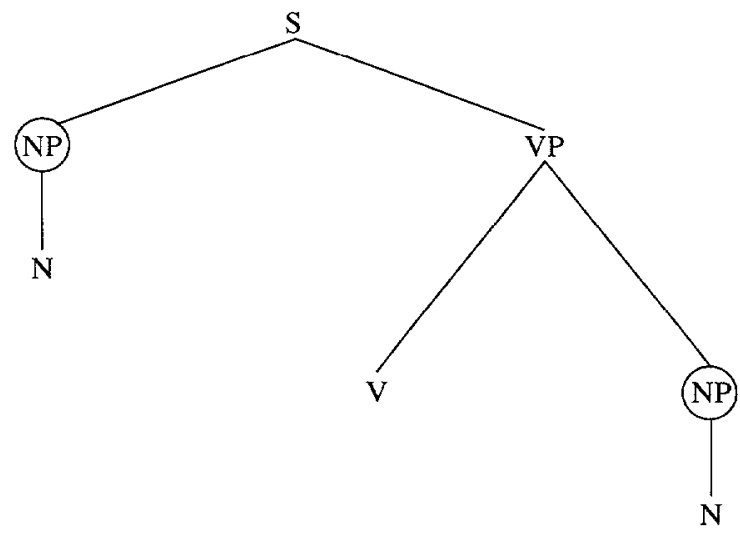

$\mathrm{S}=$ Sentence $\mathrm{NP}=$ Noun Phrase $; \mathrm{VP}=$ Verb Phrase $; \mathrm{N}=$ Noun; $\mathrm{V}=$ Verb

Figure 5

The base P-marker is converted into a final P-marker by the operation of one or more transformational rules. The final P-marker constitutes the surface structure of the sentence (Jacobsen 1977: 78).

The kernel constructions (sentences) in any language, according to Nida (1964: 66), are "the minimal number of structures from which the rest can be most efficiently and relevantly derived." Although they are not identical in all languages, "there appear to be many more parallelisms between kernel structures than between the more elaborated transforms." They are actually "deep structures" underlying the surface structures of sen- 
tences. "Kernel sentence" is a term employed in the early theories of transformational grammar but no longer used due to its incapability of solving the transformation problems of complex sentences 3 . This fact does not necessarily imply that the term "kernel" should not be employed again in a theory of translation. However, if the hypothesis has been justified, the use of the concept "kernel" in this three-stage procedure appears to be inappropriate; working on the basis of transformational grammar, this procedure will inevitably encounter the problems of transforming complex sentences.

In the Standard Theory, it is asserted that deep structure determines the meaning of a sentence while surface structure determines the form or shape of a sentence ( $O$ 'Neil 1981: 11). The deep structure contains all the information about the semantic meaning which, once determined by the deep structure, will not be altered in the process of transformations. The surface structure is only the derivation of a particular deep structure and it forms the input to the language acquisition device. Objections are made to this assertion and many facts are provided to show that semantic interpretation is not only relevant to the deep structure, but also to the surface structure. For instance, the different position of a negative word in the surface structure, particularly in conjunction with a quantity word, will alter the meaning of the sentence. To illustrate this point, Jackendoff (cited in Hou 1982a: 63-65; $c f$. Hou 1982b: 82) provides a pair of examples:

(1) $\left\{\begin{array}{l}\text { (a) Not many arrows hit the target. } \\ \text { (b) Many arrows didn't hit the target. }\end{array}\right.$

In (a), the quantity word "many" is in the scope of negation whereas in (b) "many" stays out of it. Alternatively, in (a) the scope of negation covers the whole sentence, but in (b) it covers only part of it. Hence "a left-to-right order principle" is set up (Jacobsen 1977: 468):

if a quantifier occurs to the right of "not" in surface structure, it falls within the scope of the negation. Conversely, if "not" occurs to the right of a quantifier, it falls within the scope of the quantifier ${ }^{4}$.

For example (Jacobsen 1977: 468):

(2)

$\left\{\begin{array}{l}\text { (a) He didn't see many girls. } \\ \text { (b) Many girls he didn't see }\end{array}\right.$

(3) $\left\{\begin{array}{l}\text { (a) Many students admire few lecturers. } \\ \text { (b) Few lecturers are admired by many students. }\end{array}\right.$

This principle only works on the surface-structure level. In view of this, the postulation that semantic interpretation is entirely determined by the deep structure is not verifiable (cf. Hou 1982a: 63-65; Hou 1982b: 82).

Many linguists objected to the Standard Theory which underwent a revision, emerging as Extended Standard Theory. The Extended Standard Theory introduces new concepts and terms such as "shallow structure" and "trace." In the Standard Theory, the deep structure reaches the surface structure through the process of transformation. For example, when the deep structure "you want what," becomes, by transformation, the surface structure "what do you want," the thematic relation explicitly expressed in the deep structure (what as the object of the verb want) becomes obscured by transposition in the surface structure. In the Extended Standard Theory, the place of the transposed element is marked by the trace marker $t$, for example: 


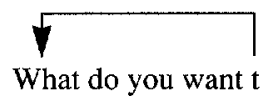

Figure 6

The structure with a trace marker $t$ is called "shallow structure." The shallow structure is a structure that comes between the deep structure and the surface structure. In the shallow structure, the thematic relation of the deep structure can be clearly traced and the concrete form of the surface takes shape; the shallow structure can accomplish the task of entire semantic interpretation. Consequently, in the Extended Standard Theory, the deep structure is irrelevant to semantic interpretation (Hou 1982a: 66) ${ }^{5}$.

The concept of "deep structure" may be useful to remove ambiguities from the relations contained in the surface structure. In the deep structure, they are explicit, for what is covert in the surface structure becomes overt in the deep structure. But deep structure cannot be expected to determine the meaning of the sentence, as has been illustrated above. Meanings expressed by the deep structure can be expressed, overtly or covertly, by the surface structure. Conversely, expressions in the surface structure may not be reproduced in the deep structure, especially in the ranges of negation and of quantity. The surface structure is the source of all the meanings that a sentence is capable of conveying $^{6}$, and so it frequently appears to be overloaded, giving rise to ambiguity. Unwelcome as it is, ambiguity is evidence that surface structure holds a great deal more than the deep structure. The failure of the surface structure to indicate certain relations openly does not imply that it does not contain them. In fact, it does and this is why a correct interpretation can be made in almost all cases, however ambiguous the surface structure may be; in reality the problem of ambiguity can nearly always be solved.

In addition, every surface structure (utterance) occurs in a particular context, either linguistic or non-linguistic. This accounts for its potential to be correctly understood. It is not in isolation as transformational grammar supposes ${ }^{7}$, but it is related in one way or another - be it ever so slightly - to the situation in which it occurs. It is not essential for all the contents of each surface structure to be totally explicit, since each one combines with other factors - including the overall situation - to clarify its meaning. If it overtly marks every semantic feature, the problem of tautology will unavoidably arise. The characteristic of ambiguity cannot be claimed to detract from the postulation that the surface structure expresses and determines the full meaning of a sentence. It only seems so, when it is seen in isolation, and artificially divorced from other surface structures to which it is situationally related.

Linguists who share the views of Miller support his argument that "complete comprehension of each part of each sentence in a communication can be attained only by transforming the surface grammatical structures into the underlying semantic or deep structures" (see Luria 1981: 171). It seems that this type of comprehension is not concerned with social and linguistic context. Even if complete comprehension as such is possible, it cannot be attained solely by proceeding from the surface to the depth. For the full meaning of a sentence to be understood, transformation from the surface to the depth must be accompanied by a careful examination of the surface structure with reference to its relations with the deep structure which have been revealed by the transforming process. If complete comprehension can only be reached by transformation from the surface to the depth, the end of this process should not be in the deep structure but it must return to the surface structure. The purpose of going to the depth is to make explicit what is unclear on the surface and ultimately to improve understanding of the surface structure. Even without looking at the deep structure, the surface structure of a sentence may be 
understood by its context (which is a broader surface structure, at least in its linguistic sense). To abandon the surface structure, however, is to abandon everything for, without the surface structure, where is the deep structure? Although, theoretically, the surface is built on the depth, in reality there is only the surface structure. Deep structure is only theoretically derived from surface structure, but this fact appears to be ignored by linguists. Deep structure is an abstraction of surface structure; the latter is the source of the former while the former presupposes the latter.

The trace theory is a technique of semantic interpretation that involves an effort of return to the surface structure. The term "shallow structure" is applied to the surface structure with a trace marker, which is directly related to semantic representation ( $c f$. Hou 1982b: 88). By availing itself of the deep structure, the trace theory marks the place on the surface left unclear by the transposed element. It relates the deep structure directly to the surface structure, linking them together with the stress on the latter. It is efficient and powerful in semantic interpretation because it works on the axis of the surface structure. The trace theory demonstrates that complete comprehension of a sentence can only be attained at the surface level when due consideration is paid to the deep structure.

Since deep structure cannot determine the meaning of a sentence, and since complete comprehension cannot be attained at the deep structure level, it must be asked whether it is appropriate to transfer from one language to another at the deep structure level or at a near-kernel level ${ }^{8}$.

\section{Transformation involves meaning-changing}

Transformation in generative grammar is a grammatical means by which surface structure is generated from deep structure. It is an essential part of the process of generating sentences, with sequential transforming rules operating between linguistic structures (see Hou 1982b: 72).

Before the Extended Standard Theory, some linguists assumed that transformations were "meaning-preserving" (see Jacobsen 1977: 93). Semantic interpretation was determined exclusively by deep structure and, once determined, it should never be altered during the process of transformation. In this view, surface structure is merely a framework carrying the input to the receptor.

This hypothesis is challenged by problems of negation and quantification. Deep structure cannot determine the scope of negation and quantification, which is finally determined by the positions occupied by the relevant negative and quantifier in the surface structure. This suggests that the meaning of a sentence may change during the transforming process. In other words, not all the transformations, particularly when negation and quantification are involved, can preserve the meaning of a sentence ( $c f$. O'Neil 1981: 46; Jacobsen 1977: 91). For example, when a sentence is converted from active voice to passive voice, its meaning generally remains unaltered. This is not the case when quantification is concerned:

(4) (a) Everybody in the room knows four languages.

(b) Four languages are known by everybody in the room.

From (a) it is understood that everybody in the room knows four particular languages (which may be as diverse as English, German, French and Spanish in one case and Chinese, Vietnamese, Italian and Japanese in another). The meaning of (b) is that four particular languages are being referred to and everybody in the room knows those four languages. So, when (a) is transformed to (b), the meaning is changed (O'Neil 1981: 125).

No two divergent surface structures derived from the same deep structure bear exactly the same meaning, taking thematic relation, presupposition, and other factors into 
consideration $^{9}$. As the Extended Standard Theory develops from its predecessor, the KPhypothesis ${ }^{10}$ is abandoned. Transformations are "allowed" to change meaning (see Jacobsen 1977: 468). To acknowledge the fact that transformations involve meaningchanging is a progressive step in the development of transformational-generative grammar. A recent claim is that transformations themselves contain semantic elements in some cases:

As a means of searching for the equivalent relations between two forms, transformations include semantic elements to a certain extent. Regularization of formal classification indicates to a considerable extent the regularization of implication interpretation (Zheng 1985: 74).

Even if transformations in the theory of transformational grammar are devoid of semantic elements, even if they really do not cause any meaning-changing, those (backtransformations and forward-transformations) applied in Nida's three-stage procedure are the processes of meaning-reducing and meaning-increasing. His back-transformations are definitely not employed to generate sentences, nor to convert sentences from one form into another; they are employed to simplify sentence structures, reducing them to their underlying kernels so that it is easier to transfer them to the receptor language. Such processes of structure-reducing will inevitably involve loss of meaning and removal of grammatical relations, for the reduced structures or the kernels are almost the skeletons of any fully-fledged surface structures.

Isolated kernels (basic structures), like isolated words, do not possess much meaning. Only when they are connected in a given combination, can they gain considerably more significance. This accounts for the wide variation in the entropy of a sentence which increases when the sentence is in a particular context and lessens when it is in isolation. Sometimes a sentence meaning in a particular context may differ greatly from its message when in isolation. Connections between and within sentences are imbued with grammatical significance, and this significance always qualifies, adjusts and increases the semantic meaning contained in those sentences. When they are reduced to kernels many of the connections will be obscured or even severed and this disruption is likely to reduce or even alter the meaning originally expressed by the surface structure.

Nida claims that transfer can be made easily at a near-kernel level because languages appear to agree far more in the depth than on the surface. This is true. When a surface structure is pruned away to a kernel, much grammatical significance and a certain amount of semantic meaning are lost. The smaller the amount of information, the easier the transfer. The entropy of information is in inverse proportion to the degree of translatability. Since the kernels are "half a dozen or a dozen basic structures" where more ostensive parallelisms are found among languages, they are certainly easy to transfer. And, since back-transformation is a structure-pruning process which involves meaning-reduction, it certainly makes the transfer easier on that level.

However, the emphasis on ease of transfer should not hide the fact that this method does not incur the minimum of distortion.

Back-transformation prunes away surface structures and consequently reduces their meanings, so the reduced structures can at best bear only part of the meaning held in the surface structures.

Also, in the stage of restructuring, forward-transformation amplifies or branches out the transferred kernels. Like back-transformation, it is not used to generate sentences, nor to convert them from one form into another. It is used here only to make the transferred materials "fully acceptable in the receptor language" (Nida 1964: 184-191; 68-69); it makes them intelligible to the target language receptor. It is not used in a strict sense of transformational grammar; it has become a technique of transfer in the three-stage procedure. It is a process of structure amplification and a process of meaning multiplication. 
The first stage is a process of structure-meaning reduction and the third is a process of structure-meaning amplification. If the amplified material is exactly the same as that which was reduced at the earlier stage, then the transfer has been made with a minimum of distortion. Unfortunately, it is generally not so.

Languages differ far more at the surface level than at the deep level; most linguistic distinctions are on the surface. When a surface structure is reduced to a kernel, many of those linguistic distinctions are necessarily lost. In the most profound deep structure no language boundaries can be found, so it is easier to transfer in the depth than on the surface. Since linguistic distinctions particular to a given language are meaning-bearing they should not be neglected in any translation. If no effort is made to represent or describe them in the target language, there is no guarantee that the rendering will be equivalent to the original. The word order, as a feature of surface structure, is an essential part of Kafka's writing, for example. To change it is to change the meaning of the original. As no two languages are identical, alteration is frequently unavoidable but it should be confined to a reasonable degree. Transfer made at a deep-structure level causes greater distortion, for it tries to avoid linguistic differences, which is impossible. To translate is to overcome such differences.

However, transformational-generative grammar is very powerful and it needs to be constrained so that only grammatical sentences are produced. Yet how can it be constrained? Jacobsen (1977: 207) points out:

Base P-markers form the input to the transformational component and final P-markers form the output. It is therefore between these two poles that the constraints must be placed.

In fact, the process of transformation must be constrained. The state of being grammatical is always language-specific, so the process of transformation is constrained differently in different languages. It may follow the same T-rules in all the languages but each method may be idiosyncratic.

Nida's back-transformations and forward-transformations have been shown as techniques used in the transfer procedure to reduce and extend language structures. Even if the inference concerning the process of transformation is untenable, these two techniques rnust conform to the grammars of the given languages in which they are carried out, especially the forward-transformation which is intended to make the transferred material fully acceptable to the TL receptor. The obvious reason is that for a sentence to be acceptable grammaticalness must be presupposed, and, in turn, this presupposes conformation to the grammar of the language concerned.

In translation, the movement from surface to depth in a source language and then from depth to surface in a target language are two separate processes of transformation in reverse directions, and they are connected through the enigmatic transfer. How can these two processes be constrained? In the first process the rules of the source language must be observed while in the second those of the target language must be followed, but these rules can only guarantee that no deep or surface structures would appear that violate the norms of the languages concerned. It is unlikely that these rules will allow transformation to preserve the meaning during its process. These two processes reduce and amplify the meaning of the data being transformed and since each reduces or amplifies in its own way, there is no guarantee that the target language surface structure will correspond semantically to the source language deep structure; chance of correspondence between the two surface structures is consequently more remote. To obtain semantic correspondence between the SL and TL surface structures, which is the principal motivation of this three-stage procedure - in fact, the goal of any translation method - it is essential to go back and compare the two surface structures, readjusting the construction of the TL 
surface to make it more compatible with that of the SL surface in either meaning or function - or in both.

The paradox here is that in order to achieve equivalent response, the TL surface structure must be compared to that of the SL, for equivalent response can only be elicited by the equivalence held between the SL and TL surface structures in terms of construction, meaning or function. As the surface structure is difficult to transfer, owing to its wealth of meaning and linguistic distinctions, it seems preferable to transfer at the deep level. However, when transfer is made at the deep-structure level or the near-kernel level, no compatibility between the SL and TL surface structures can be guaranteed, for no parallelism can be secured between the two processes of transformation in the two languages concerned $^{11}$. Consequently, no equivalent response can be guaranteed.

Nida (1969b: 104; 39-40 footnote) suggests that when the kernel level is reached, the translator should then back up a little so as not to obscure the connections between the kernels. This movement permits more accurate meaning, but makes transfer more difficult. It is an expedient employed to make transfer slightly easier by going from surface to depth and to make the TL surface structure a little more compatible with that of the SL by backing up a little in the architecture of linguistic structure. This method cannot solve the problem satisfactorily for the ease of transfer and the compatibility between the SL and TL surface structures stand in a contradictory relationship where the choice of one involves abandonment of the other. Theoretically, it appears inadvisable to follow the three-stage procedure, and it is doubtful whether it is actually used in practical translation.

This discussion has demonstrated that the compatibility between the SL and TL surface structures should not be neglected. Such compatibility is the base on which a type of equivalence is set up. Therefore, the putative equivalent response finally depends on such compatibility. This being so, the structuring of the TL surface should be based on that of the SL surface structure. That is, it should be structured to approximate closely to the SL surface structure in terms of construction, meaning or function. Although unable to "represent accurately the procedure of good translation," the single-stage procedure at least implies the direct comparison of the TL surface structure to the SL surface structure which is indispensable to any kind of translating procedure.

In his essay Deep Structure, Surface Structure and Translation, Humberto Lopez Morales (1982: 20) argues that the theory of transformational-generative grammar will help the translator to free himself from the influence of the source language and to transfer the meaning of the source language from its deep structure to that of the receptor language and then to arrive at the receptor language surface structure by means of transformation. Also, the application of this theory in translation will aid comprehension of the source language and assist in solving problems of source language structure ambiguities. This clearly overestimates the function of transformational grammar theory in the translating process. Transformations will not be particularly useful in understanding the source language and solving its structural ambiguities unless it returns to its surface structure. The best transfer should be that which can procure a high degree of compatibility between the TL surface structure and the SL surface structure in terms of construction, meaning of function. The application of the transformational-generative theory in translation cannot guarantee such compatibility. It is employed only to avoid the difficulties caused by linguistic differences, and may lead to greater distortion, which then runs counter to the motivation for applying the transformational grammar theory in the translating procedure. In addition, to translate is not to evade the difficulties the translator is bound to encounter during the translating procedure, but to overcome them in order to seek an acceptable correspondence between the differences. 


\section{About transfer}

How does man stop thinking in one language and begin thinking in another? This is still a mystery, so Nida (1982: 51) admits that the description of any translation process cannot be comprehensive. This may explain why he postulates a "transfer stage" without detailing the process ( $c f$. Kelly 1979:36).

Although transfer as a mental process remains a mystery, hypotheses have been formed in an attempt to describe it. A Chinese translation theorist (Yang 1984: 297) assumes that it is a process during which the meaning elements of the source language enter the human brain and they are then rearranged in accordance with the norms of the target language. This assumption bears some resemblance to the single-stage procedure as, in both the processes, the two languages involved contact directly, without making any detour.

Walmsley (cf. Kelly 1979: 43) designs a three-step procedure, similar to Nida's three-stage procedure:

First, the text is to be rewritten in the source language using sentence patterns of the target language in an effort to isolate the deep structure. Then, the text is to be rewritten in the target language following the first structures exactly. Finally, the complete text is to be stylistically reconstructed by applying appropriate tranformations.

Although this procedure also adopts the theory of transformational grammar, it does not rigorously pass through two processes of transformation. It translates little by little and step by step; transformation is only one step in the process. In Nida's method, transformational grammar serves as a definite theoretical framework and transformations as techniques run through almost the whole procedure. But Walmsley involves a transplantation reminiscent of "the French seventeenth-century idea of redrafting texts in l'ordre naturel before producing a finished text from them" (Kelly 1979: 43).

Although Nida does not describe the transfer itself in detail, he quotes a three-stage translation analysis devised by William Wonderly (1964: 184-191). This technique is originally designed for classifying translations into various categories, but it also presents an image of the process of transfer. The first stage is literal transfer, where a source language text is copied without any alteration, using receptor language symbols; the second stage is minimal transfer, where any necessary change - the smaller, the better - is made to allow the rendering to be intelligible; the final stage is literary transfer, where readjustments are made to create idiom and style. Alteration at the third step is optional and translations of a given text diverge from this step.

This method is unlike the three-stage procedure. There is no impact of transformational grammar and the transfer is made at the surface-structure level from beginning to end. Ironically it bears a strong resemblance to Walmsley's three-step procedure about which Kelly (1979: 43) writes: "Whether it adds anything to traditional views of translation as transmission is very doubtful."

In Bassnett-McGuire (1980: 17), another postulation is made:

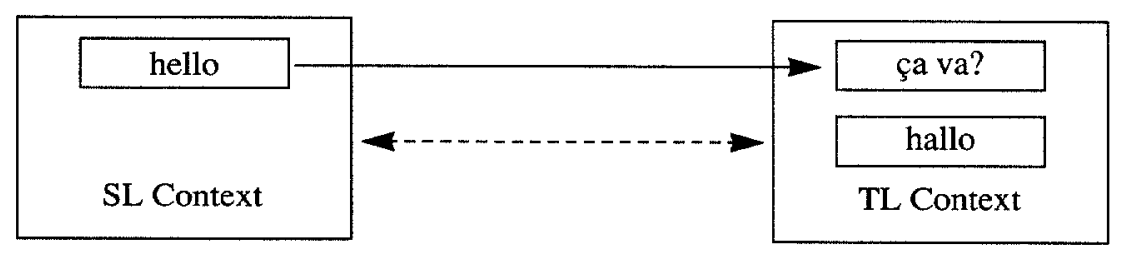

Figure 7 
This diagram illustrates the way in which the English word "hello" is put into French. There are two French expressions that correspond to "hello:" Ça va? and hallo. But this does not present too much difficulty, for "hello" is always used in a particular situation or context. The particular context definitely determines its exact meaning, and indicates its function in the communication. The translator/interpreter, therefore, can directly make a choice between "ça va?" and "hallo" on the basis of the French usage in the same situation or context. This process follows a straight route, like the single-stage procedure. It seems better than the others, for it takes nonlinguistic factors into account, including social and cultural features.

Each of these theories presents an overly simple image of transfer as a mental or psychological process. In actual translation, the activities of the human brain are complex and the linguistic data to be processed will not be so "pure" and treatable. However, to a degree they have shed a faint light on the problem of transfer.

It can be concluded that transfer as a mental process demands a comparison of the TL surface structure with the SL surface structure in terms of construction, meaning or function, for all equivalence is based on the compatibility between these three areas in the two surface structures. An acceptable correspondence between the two surface structures is the aim of every translation and a good transfer model should be able to achieve such compatibility. Also, it is better to transfer at the surface-structure level, as transformations involve distortion of meaning. If transfer is made on a level below the surface structure, attention should always be paid to a comparison between the two surface structures since surface structure contains all the expressed meaning.

\section{Notes}

1. Other techniques, such as componential analysis, are also employed at this stage to analyze the meanings of the words and combinations of words. As any translation involves analysis of grammatical relationships and word meanings, it is the way in which translation is done which is important. In his procedure, the analysis of word meanings, though important, is not typical of the three-stage system, which adopts the theoretical framework of transformational grammar. So comment on these techniques is omitted due to the limitations of this paper in length.

2. Cf. Chomsky, N.: "Recent Contributions to the Theory of Innate Ideas," J.R. Searle (ed.), The Philosophy of Language, p. 125: "Deep structures seem to be very similar from language to language."

3. S.N. Sridhar (1981: 216): "Dingwall proposed that languages are more likely to be similar in their kernel than in their total structure, and that which is obligatory in the most valued grammar is more basic than that which is optional," but with the demise of the notion of kernel sentences, his hypothesis has become somewhat outdated.

4. Jacobsen (1977: 468): "The exception to the principle is All that glitters is not gold." Cf. Ji G.Q. (1982: 194-195).

5. However, there are some linguists who still affirm that deep structure determines the meaning of a sentence. $C f$. Hou (1982b: 82-83): "They bind semantic representation up with deep structure far more closely, even to the extent that semantic representation and deep structure become merged into a single whole. And this is generative semantics. It strives not only to take into consideration the part that surface structure plays in determining semantic representation, but also sticks to the identity of deep structure and semantic representation, thus introducing the concept of global rules. These are a group of rules which are intended to relate the abstract semantic representation in the deep structure to those characteristics of the surface structure that determine the meaning of the sentence. When generative semanticists start to introduce into grammar such non-linguistic elements as belief and attitude, things become even worse. For this has really deviated from the real aim of studying linguistic theory - to explain the phenomenon of language acquisition.

6. $C f$. Hou (1982b: 86): "With the development of the trace theory, we can say that all the semantic representations, including thematic relation, can be in a way deduced from the surface structure." $C f$. also Ji G.Q. (1982: 198): "If theme, centre and presupposition are taken into consideration, it is almost impossible for any two sentences which share the same deep structure but are embodied in different surface structures to be synonymous." - If this is true, it can be postulated that the surface structure ultimately determines the meaning of a sentence. 
7. Cf. Steiner (1975: 108). He quotes George Lakoff, saying: “...it [transformational grammar] tried to study the structure of language without taking into account the fact that language is used by human beings to communicate in a social context."

8. Nida (1969b: 39-40) claims that transfer is made on a near-kernel level which is above the deep structure. This near-kemel level, however, must be rather deep in the architecture of linguistic structure, for in such a procedure, all the sentences in a language must be reduced in terms of structure to half a dozen kernels, which can only be realized on a very deep level. So we still consider this "near-kernel level" as a sort of deep-structure level, though with the awarness that it is claimed to be somewhat higher than the deep structure in the normal sense.

9. Cf. note 6 .

10. The KP-hypothesis was devised by J.J. Katz and P.M. Postal; it postulates that deep structures fully determine meaning. For a discussion of the KP-hypothesis, see Fodor (1977: 64-70).

11. Cf. Carol Sanders (1981: 25): “...his [the mature learner] mistake lies in assuming that the target language surface structures will be the same as in his L1, that is, that the deep to surface structure procedure will be the same." Nida's application of transformational theory in translation also seems to take for granted the parallelism between the back-transformation procedure in a source language and the forward-transformation procedure in a receptor language. 